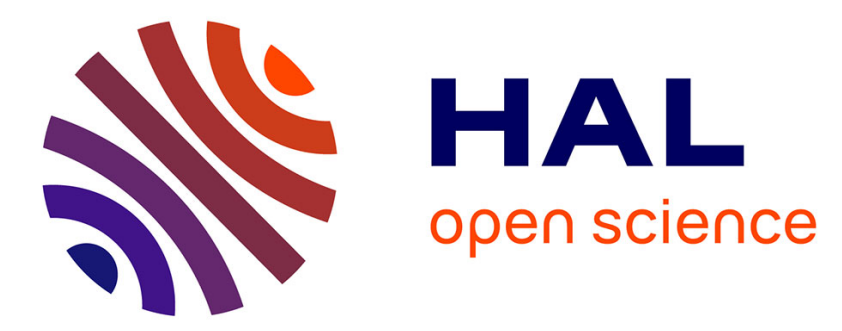

\title{
Traitement de la douleur du sujet âgé
}

Arielle Simunek, Serge Perrot, Anne-Priscille Trouvin

\section{To cite this version:}

Arielle Simunek, Serge Perrot, Anne-Priscille Trouvin. Traitement de la douleur du sujet âgé. Revue du Rhumatisme monographies, 2019, 86, pp.228 - 232. 10.1016/j.monrhu.2019.03.003 . hal-03484442

\section{HAL Id: hal-03484442 \\ https://hal.science/hal-03484442}

Submitted on 20 Dec 2021

HAL is a multi-disciplinary open access archive for the deposit and dissemination of scientific research documents, whether they are published or not. The documents may come from teaching and research institutions in France or abroad, or from public or private research centers.
L'archive ouverte pluridisciplinaire HAL, est destinée au dépôt et à la diffusion de documents scientifiques de niveau recherche, publiés ou non, émanant des établissements d'enseignement et de recherche français ou étrangers, des laboratoires publics ou privés.

\section{(ㄷ)(1) $\$$}

Distributed under a Creative Commons Attribution - NonCommercial| 4.0 International 


\section{Revue du Rhumatisme Monographie}

Traitement de la douleur du sujet âgé.

Treatment of pain in the elderly

Arielle SIMUNEK ${ }^{1}$, Serge PERROT ${ }^{1,2}$, Anne-Priscille TROUVIN ${ }^{2,3}$

1. Centre d'Evaluation et Traitement de la Douleur, AP-HP Hôpital Cochin, Université Paris Descartes ; 27 rue du Faubourg Saint Jacques, 75014 Paris ; France

2. Inserm U987 Physiopathologie et Pharmacologie Clinique de la douleur, Hôpital Ambroise Paré ; 9 Avenue Charles de Gaulles, 92100 Boulogne Billancourt ; France

3. Centre d'Evaluation et Traitement de la Douleur, AP-HP Hôpital Ambroise Paré ; 9 Avenue Charles de Gaulles, 92100 Boulogne Billancourt ; France

\section{Auteur Correspondant}

Anne-Priscille Trouvin

Centre d'Evaluation et Traitement de la Douleur

INSERM U987

Hôpital Ambroise Paré

9 avenue Charles de Gaulle

92100 Boulogne Billancourt

Téléphone : + 33149095946

Fax : + 33149094435

Mail : annepriscille.trouvin@aphp.fr

Mots clés : gériatrie, antalgiques, douleurs musculo-squelettiques, sujet âgé, traitement non pharmacologique

Keywords: elderly, analgesics, musculoskeletal pain, geriatric pain, non-pharmacological treatment 


\section{Résumé}

La douleur du sujet âgé est sous-évaluée et difficile à prendre en charge en raison des multiples comorbidités et interactions médicamenteuses spécifiques à ces patients.

Si plusieurs recommandations internationales existent pour la gestion du traitement de la douleur chez le patient âgé, un consensus français, multidisciplinaire d'experts en douleur et gériatrie a été publié en 2017 pour guider les prescriptions.

Sur le plan pharmacologique, les antalgiques sont utilisables chez le patient âgé en respectant certaines précautions. II faudra principalement introduire les traitements un à un afin de surveiller leur efficacité propre et leur tolérance, il conviendra d'adapter les posologies à l'âge, les insuffisances d'organes et surtout les co-prescriptions.

Afin d'optimiser le traitement l'association de thérapeutiques non médicamenteuses doit être envisagée et proposée. Enfin pour favoriser l'observance il conviendra d'expliquer au patient et à son entourage le traitement mis en place, son bénéfice attendu, ses effets indésirables potentiels.

\section{Abstract}

The elderly patient's pain is undervalued and difficult to manage because of the multiple comorbidities and drug interactions specific.

While several international recommendations exist for the management of pain management in the elderly patient, a French multidisciplinary consensus of experts in pain and geriatrics was published in 2017.

Analgesics can be used in elderly patients by following certain precautions. It will mainly be necessary to introduce the treatments one by one in order to monitor their own efficacy and their tolerance, it will be necessary to adapt the dosages to: the age, hepatic or renal failure and especially the coprescriptions.

In order to optimize the treatment, the combination of non-pharmacological therapies must be considered and proposed. Finally, to promote adherence, it will be important to explain to the patient and his entourage the treatment put in place, its expected benefit, and its potential adverse effects. 


\section{Manuscrit}

\section{Introduction}

La prévalence de la douleur chronique quotidienne est estimée à $31 \%$ en population générale (1). Dans l'étude STOPNET, l'enquête en population générale française a montré une augmentation statistiquement significative de cette prévalence de la douleur chronique avec l'âge, elle atteint $52,4 \%$ dans la population d'âge supérieur à 75 ans (1). Ce chiffre est comparable à d'autres pays tel que les Etats-Unis (2). La prévalence de la douleur peut atteindre plus de $90 \%$ chez les patients âgés institutionnalisés (3).

La douleur de la personne âgée est généralement sous-évaluée. Certaines hypothèses ont été formulées concernant la problématique de l'évaluation de la douleur par ces patients. La plainte peut être non spécifique ; en cas de troubles cognitifs, les patients peuvent avoir une limitation dans leur capacité à caractériser leurs sensations voire être dans l'incapacité de verbaliser leur plainte (4).

Les plaintes douloureuses des patients âgés sont principalement les douleurs lombaires, les douleurs musculosquelettiques, les douleurs neuropathiques et les douleurs articulaires périphériques (5). Les échelles et questionnaires d'évaluation de la douleur ne sont pas toujours adaptés à la population gériatrique. Une revue de la littérature a montré que les échelles les plus performantes, pour les patients âgés sans trouble cognitif, sont : l'échelle verbale simple, l'échelle numérique, le Brief Pain Inventory (traduit en français : Questionnaire Concis de la Douleur) et le Geriatric Pain Measure (6). Chez les patients ayant des troubles cognitifs ou non communiquant deux échelles spécifiques de la population gériatriques existent : l'échelle DOLOPLUS et l'échelle ALGOPLUS $(7,8)$. Ce temps d'évaluation de la douleur est un préalable nécessaire avant toute introduction d'un traitement. Une évaluation précise de la douleur au-delà de la simple intensité est nécessaire, il faudra ainsi évaluer en outre, les caractéristiques de la douleur telles que sa localisation (localisée ou diffuse), son rythme (mécanique, inflammatoire, permanente), le caractère nociceptif ou neuropathique, ses répercussions fonctionnelles et donc son impact sur la vie quotidienne et l'autonomie du patient.

Au-delà de la prévalence importante de la douleur chez la personne âgée, des difficultés éventuelles pour ces patients à exprimer leur plainte et enfin des limites de l'évaluation du symptôme douloureux en population gériatrique, le dernier problème est celui de l'adaptation thérapeutique. Chez le patient âgé, il existe un risque majoré d'effets indésirables des traitements en raison des changements physiologiques liés à l'âge qui modifient la pharmacocinétique et la pharmacodynamique des médicaments, ainsi que de multiples morbidités et les interactions médicamenteuses parfois nombreuses (9).

\section{Prise en charge pharmacologique}

Plusieurs recommandations ont été publiées au niveau international (10 - 12). En France, un consensus multidisciplinaire d'expert a été publié en 2017 (13) basé sur l'analyse des recommandations internationales, sur les résumés des caractéristiques des produits et sur l'avis des experts.

Les recommandations retenues dans le consensus sont les suivantes (13): 
- Concernant le paracétamol : "le paracétamol demeure le médicament prescrit en première intention dans les douleurs faibles à modérées. Toutefois un ajustement posologique est recommandé dans un contexte de comorbidités et de co-prescription de médicaments anticoagulants à la posologie de $3 \mathrm{~g}$ par jour chez le patient de plus de 65-70 ans, en fonction de son état de santé." (13)

Concernant l'interaction avec les anticoagulants, une méta-analyse a montré un risque significatif d'augmentation de l'INR chez les patients recevant du paracétamol en co-prescription avec un anti-vitamine $\mathrm{K}(14)$.

- Concernant les anti-inflammatoires non stéroïdiens : "le profil médical du patient est évalué initialement et réévalué périodiquement. La prescription d'un AINS est envisagée seulement après échec du paracétamol. La posologie doit être la plus faible possible sur la durée la plus courte de l'ordre de quelques jours (< 8 jours) en faisant un contrôle de la créatininémie à j5. Une association à un inhibiteur de la pompe à protons est indispensable. Une évaluation régulière de la prescription est nécessaire pour s'assurer que la balance bénéfice/risque reste positive en recherchant les effets indésirables et les interactions médicamenteuses potentiellement néfastes. II ne faut pas administrer deux AINS ou dépasser la dose efficace. Les AINS topiques sont une très bonne alternative thérapeutique dans l'arthrose du genou et de la main. Il est déconseillé de les associer à un certain nombre de médicaments qui sont largement présents chez la personne âgée (AVK, nouveaux anticoagulants oraux, IEC/ARA 2+ diurétiques...). Il est important de s'assurer de la bonne compréhension du traitement, en particulier les génériques, et de vérifier l'automédication." (13)

Concernant le choix de la molécule, il conviendra de ne pas prescrire de molécule à demi vie longue telles que le naproxène, sulindac, et oxicams. Concernant le naproxene, il présente un profil de sécurité supérieur aux autres molécules chez les patients à risque cardio-vasculaire (15) cependant les études de pharmacocinétique chez le sujet âgé montrent une augmentation de la fraction libre du médicament, il conviendra donc en cas de prescription de diminuer la dose prescrite $(16,17)$

Les données de la littérature montrent une augmentation significative des effets indésirables rapportés lors de l'usage d'AINS chez les patients âgés (18). Concernant le choix spécifique d'un AINS inhibiteur de la COX2, une large étude cas-témoins montre une diminution significative du risque digestif lors de l'association AINS et inhibiteur de la pompe à protons comparé au groupe de patient de moins de 75 ans. En revanche dans le groupe âgé de plus de 75 ans, le risque d'effet indésirable digestif lors de l'utilisation de COX-2 sans inhibiteur de la pompe à protons était supérieur comparé aux patients jeunes (19). Chez le sujet âgés il conviendra donc d'associer systématique un inhibiteur de la pompe à protons quelle que soit la molécule AINS prescrite.

- Concernant le néfopam : Il s'agit d'un antalgique d'action centrale non dérivé opioïde. II convient de rappeler que son indication est limitée aux douleurs aigues notamment postopératoire. La forme galénique disponible en France est la forme injectable. Le dernier avis de commission de transparence rappelle qu'il ne doit pas être utilisé dans la prise en charge des douleurs chroniques et qu'il convient de ne pas l'utiliser de façon détournée par voie 
orale (20). Les contre-indications de cette molécule sont les convulsions ou antécédents de troubles convulsifs, les risques de rétention urinaire liés à des troubles urétroprostatiques et les risques de glaucome par fermeture de l'angle (13).

- Concernant les opioïdes faibles : "Les opioïdes faibles ont toute leur place dans le traitement de la douleur aiguë et chronique de la personne âgée. La multiplicité des associations fixes paracétamol-opioïdes faibles doit être bien connues ; leur prescription doit être rigoureuse et la posologie de l'opioïde spécifié. Leur initiation à dose réduite et leur augmentation progressive sont rendues possibles par certaines formes galéniques qui offrent de possibilités d'adaptation posologique en étant vigilant sur les problèmes de vue et de tremblement. Le tramadol est la molécule de choix en cas de douleur mixte [association d'une douleur par excès de nociception et d'une douleur neuropathique]."(13)

Si l'efficacité des molécules opioïdes faibles est comparable entre les molécules le risque de survenue des effets indésirables l'est aussi. Ces derniers sont favorisés chez les patients âgés par des posologies recommandées non adaptées à la population âgées mais aussi le problème de variation génétique portant sur le cytochrome p450 2D6 voie de métabolisation du tramadol, de la codeine, de l'oxycodone. Outre les variations génétiques, de nombreuses autres molécules pharmacologiques font varier l'activité de ce cytochrome soit en induction soit en inhibition (anti-arythmiques, antidépresseurs...) (13).

Le tramadol est actuellement la molécule opioïde faible dont le maniement est facilité en gériatrie par l'existence d'une formulation buvable permettant ainsi une titration orale et donc l'introduction de cette molécule à dose faible et progressivement croissante comme proposé dans plusieurs publications $(21,22)$. Les formulations buvables disponibles en France ont une concentration $100 \mathrm{mg} / \mathrm{ml}$ soit $2.5 \mathrm{mg} /$ goutte ce qui permet de faire des paliers de titration intermédiaires. Chez le sujet âgé, il peut exister des erreurs de prise médicamenteuse, il convinedra donc d'être vigilant concernant ce risque en particulier en cas de trouble visuel ou de tremblements par exemple.

- Concernant les opiö̈des forts : "Il faut en pratique prévoir un traitement de fond et la possibilité de doses de "recours" en cas d'accès douloureux paroxystiques. Les posologies recommandées pour initier l'opioïde fort 15 à $30 \mathrm{mg}$ de morphine orale/jour ou 10 à $20 \mathrm{mg}$ d'oxycodone orale/jour. On choisira la dose recommandée la plus faible pour les plus fragiles." (13) " Il faut connaitre les différentes galéniques afin de tenir compte des capacités de déglutition et voies d'abord du malade. Il faut choisir la voie d'administration la moins invasive possible et privilégier la voie orale. Il faut introduire un seul antalgique/médicament à la fois pour pouvoir évaluer et attribuer précisément d'éventuels effets secondaires. La titration de la dose efficace se fait préférentiellement avec un opiacé d'action immédiate réparti toutes les 4 à 6 heures afin d'ajuster au mieux la posologie en tenant compte de l'efficacité et des effets secondaires." (13)

Les données actuelles sur la pharmacocinétique et la pharmacodynamie chez les patients âgés ne permettent pas de recommander une molécule plus qu'une autre (13). Cependant le fentanyl transdermique n'ayant pas une élimination rénale il sera à privilégier en cas d'insuffisance rénale. 
Le fentanyl n'est en revanche pas recommandé pour l'introduction d'un traitement opioïde fort (13).

Dans la douleur chronique non cancéreuse, il conviendra de respecter les recommandations de la Société Française d'Etude et de Traitement de la douleur publiées en 2016 (23). Ces recommandations rappellent la nécessité d'évaluer le risque de mésusage avant la prescription d'opioïdes forts et de réévaluer l'existence d'un possible mésusage à chaque renouvellement de traitement (23). Chez les patients âgés le risque d'addiction est inférieur au risque existant chez les patients jeunes (13).

La douleur musculo-squelettique peut présenter une composante neuropathique, les différentes études estiment par exemple que la prévalence d'une douleur neuropathique dans l'arthrose de genou ou de hanche est de $23 \%$ (24) et de $20 \%$ chez les patients ayant un rhumatisme inflammatoire (25). Ces prévalence peuvent nous amener à considérer l'utilisation des traitements pharmacologiques anti-neuropathiques cependant leur maniement peut être plus complexe que les antalgiques usuels en particulier chez les sujets âgés. Tout d'abord c'est ici la présence d'une composante neuropathique avérée évaluée par exemple avec le questionnaire DN4 (26) qui guide la prescription, l'intensité de la douleur n'est pas dans ce cas le critère de choix (13). L'efficacité de ces traitements peut mettre plusieurs semaines à s'installer, il faut donc réévaluer régulièrement les patients et expliquer cette efficacité retardée aux patients afin d'améliorer l'observance. Les recommandations internationales (27) recommandent en première intention les gabapentinoïdes (gabapentine et prégabaline), les antidépresseurs tricycliques (amitriptyline) ou les antidépresseurs inhibiteurs de recapture de la sérotonine et de la noradrénaline (duloxetine, venlafaxine). En deuxième ligne de traitement la capsaïcine en patch à haute concentration, les patchs de lidocaïne (en France leurs AMM sont limitées aux douleurs post-zoostériennes) et le tramadol (27). Enfin les opioïdes forts sont proposés en troisième ligne de traitement pour les douleurs neuropathiques (27).

L'introduction des traitements recommandés en première ligne doit se faire de façon très progressive et prudente chez les patients âgés (Encadré 1). Le profil de sécurité des trois classes pharmaceutique fera préférer en première intention les gabapentinoïdes (13) avec l'introduction de prégabaline à $25 \mathrm{mg} /$ jour ou de gabapentine à $100 \mathrm{mg} /$ jour puis une augmentation lente par paliers progressifs tous les 4 jours jusqu'à la dose minimale efficace (13). Pour les antidépresseurs inhibiteurs de recapture de la serotonine et de la noradrénaline il conviendra de commencer aux doses minimales (duloxétine $30 \mathrm{mg} / \mathrm{jour}$ ou venlafaxine $37,5 \mathrm{mg} / \mathrm{jour}$ ) puis une augmentation si besoin jusqu'à la dose minimale efficace $(13,28)$. La duloxetine est contreindiquée en cas d'insuffisance hépatique ou rénale sévère ainsi qu'en cas d'hypertension artérielle non contrôlée et ne doit pas être associée à la ciprofloxacine ou à l'énoxacine. La venlafaxine se prescrira à demi-dose en cas d'insuffisance hépatique ou rénale sévère, il sera vérifié l'absence d'hypertension artérielle non contrôlée, de risque de glaucome. Ces deux molécules peuvent aussi induire des troubles hydroélectrolytiques à type d'hyponatrémie ou l'apparition de mouvements anormaux ou tremblements (13).

- $\quad$ Concernant les traitements des douleurs neuropathiques : "Le traitement des douleurs neuropathiques est un traitement prolongé de plusieurs mois, voire d'avantage. 
L'intervention d'une infirmière, permettant d'encadrer la prise médicamenteuse et facilitant l'observance, est indispensable. Les traitements topiques sont à privilégier dans les douleurs neuropathiques localisées. L'initiation de ces traitements antalgiques nécessite une prise en charge multimodale. Les effets indésirables médicamenteux doivent être dépistés le plus tôt possible afin de maîtriser les risques iatrogènes fréquents dans ces classes thérapeutiques chez la personne âgée." (13)

\section{Prise en charge non médicamenteuse}

Compte tenu des risques mentionnés plus haut, l'intérêt pour les approches non pharmacologiques dans le traitement de la douleur chez les personnes âgées augmente (29).

L'hypnose a été essayée dans une étude randomisée contrôlée contre un bras avec massages chez des patients âgés (âge moyen 80 ans) sans trouble cognitif, hospitalisés et ayant des douleurs chroniques. Cette technique a permis une diminution significative des douleurs. L'effet s'est en revanche estompé à 3 mois de la sortie d'hospitalisation (30).

La thérapie cognitivo-comportementale a fait l'objet de plusieurs revues de la littérature. La revue de Lunde et al. en 2009 montre que les interventions cognitives et comportementales apportent un soulagement de la douleur. La taille de l'effet calculé est de 0,47 . Les auteurs notent qu'il n'y a en revanche aucun effet significatif sur les symptômes dépressif, ni sur le plan fonctionnel ou sur l'épargne médicamenteuse (31). Une nouvelle revue avec méta-analyse publiée cette année montre que les interventions psychologiques dans le traitement de la douleur chronique chez les patients âgés ont de petits effets, notamment une diminution de l'intensité douloureuse et du catastrophisme (32).

L'acupuncture a aussi été évaluée dans plusieurs revues et méta-analyse. La méta-analyse de Lin et al. démontre que l'acupuncture peut améliorer la fonction à court et à long terme dans la gonarthrose (33).En revanche cette méta-analyse montre qu'il n'existe qu'une efficacité de court terme de l'acupuncture sur la douleur (33). La revue Cochrane de 2010 conclue a une efficacité de l'acupuncture à court terme sur les douleurs d'arthrose périphérique (34). Ces bénéfices restent faible et n'atteignent pas une diminution de l'intensité douloureuse considérée comme cliniquement pertinente. L'analyse centrée sur les essais contrôlés contre liste d'attente suggère une efficacité sur la douleur statistiquement significative et cliniquement pertinente (34).

Le mindfulness (méditation pleine conscience), dans une étude randomisée contrôlée contre éducation thérapeutique, a montré une amélioration de l'intensité douloureuse à long terme et de la fonction à court terme dans la lombalgie chronique chez des patients âgés de plus de 65 ans (35).

La pratique d'exercices physique permet aussi une diminution de l'intensité douloureuse, la taille de l'effet calculée varie de 0,25 à 2,75 chez les patients âgés ayant une gonarthrose (10).

L'usage de la neurostimulation transcutanée a été évalué dans la lombalgie chronique avec stratification par groupe d'âge(36). Les patients âgés ont présenté les mêmes réponses au TENS en termes d'efficacité, cependant cette réponse était obtenue pour des amplitudes plus importantes chez les patients âgés. 
De façon générale, plusieurs recommandations simples peuvent aussi aider à la prise en charge du patient âgé douloureux (37) :

- Etre prêt à répondre aux questions et préoccupations concernant chaque médicament ou une combinaison de ceux-ci;

- Proposer au patient des traitements de recours lors des essais de nouveaux médicaments;

- Être disponible pour écouter et être réceptif aux préoccupations des patients;

- Évitez de garantir une efficacité de la prise en charge proposée

- Insister sur la nécessité de respecter la prescription et les horaires de prise, en particulier les formes à libération prolongée ou immédiate

- Encouragez le patient à appeler si de nouveaux signes ou symptômes apparaissent après le début du traitement;

- Élaborer un plan de surveillance minutieux pour déterminer si les objectifs de traitement sont atteints et pour surveiller les effets indésirables potentiels des traitements introduits

- Si les objectifs ne sont pas atteints, envisager de réduire progressivement les traitements pharmacologiques.

\section{Conclusion}

La prise en charge la douleur chez la personne âgée est complexe à bien des égards, sa détection est aléatoire, son évaluation est souvent imparfaite et enfin sa prise en charge est limitée par de multiples facteurs.

Le consensus d'expert publié en 2017 permet de guider la prescription d'antalgiques chez les patients âgés. Afin d'améliorer l'efficacité des traitements pharmacologiques les prises en charges non médicamenteuses doivent être associées en vue d'une prise en charge multimodale et avec pour objectif de limiter autant que possible les augmentations de posologies.

Conflits d'intérêts : AS ne déclare aucun conflit d'intérêt en lien avec cet article, SP déclare Serge Perrot avoir été membre de board pour Grunenthal, Mundipharma, BMS, Ethypharm., APT déclare des interventions ponctuelles pour les laboratoires Pfizer, Astellas, Menarini. 


\section{Références}

1. Bouhassira D, Lantéri-Minet M, Attal N, et al. Prevalence of chronic pain with neuropathic characteristics in the general population. Pain. 2008;136:380-7.

2. Patel KV, Guralnik JM, Dansie EJ, et al. Prevalence and Impact of Pain among Older Adults in the United States: Findings from the 2011 National Health and Aging Trends Study. Pain. 2013;154:

3. Brown ST, Kirkpatrick MK, Swanson MS et al. Pain experience of the elderly. Pain Manag Nurs. 2011;12:190-6.

4. Dentino A, Medina R, Steinberg E. Pain in the Elderly: Identification, Evaluation, and Management of Older Adults with Pain Complaints and Pain-related Symptoms. Prim Care. 2017;44:519-528.

5. Deleens R, Pickering G, Hadjiat Y. Douleur du sujet âgé et cognition : état des lieux. Geriatr Psychol Neuropsychiatr Vieil 2017;15:345-56

6. Kang Y, Demiris G. Self-report pain assessment tools for cognitively intact older adults: Integrative review. Int J Older People Nurs. 2018;13:e12170.

7. Pickering G, Gibson SJ, Serbouti S, et al. Reliability study in five languages of the translation of the pain behavioural scale Doloplus. Eur J Pain. 2010;14:545.e1-10.

8. Bonin-Guillaume $\mathrm{S}$, Jouve $\mathrm{E}$, Lauretta $\mathrm{R}$, et al. Algoplus performance to detect pain in depressed and/or demented old patients. Eur J Pain. 2016;20:1185-93.

9. Mangoni AA, Jackson SH. Age-related changes in pharmacokinetics and pharmacodynamics: basic principles and practical applications. Br J Clin Pharmacol. 2004; 57:6-14.

10. Makris UE, Abrams RC, Gurland B, et al. Management of persistent pain in the older patient: a clinical review. JAMA. 2014;312:825-36.

11. American Geriatrics Society Panel on Pharmacological Management of Persistent Pain in Older Persons. Pharmacological management of persistent pain in older persons. J Am Geriatr Soc. 2009 Aug;57(8):1331-46.

12. Abdulla A, Adams N, Bone M, et al. Guidance on the management of pain in older people. Age Ageing. 2013 Mar;42 Suppl 1:i1-57.

13. Capriz F, Chapiro S, David L. et al. Consensus multidisciplinaire d'experts en douleur et gériatrie : utilisation des antalgiques dans la prise en charge de la douleur de la personne âgée (hors anesthésie). Douleurs Evaluation - Diagnostic - Traitement 2017;18:234-247.

14. Caldeira D, Costa J, Barra M, et al. How safe is acetaminophen use in patients treated with vitamin K antagonists? A systematic review and meta-analysis. Thromb Res. 2015;135:58-61.

15. Schmidt M, Lamberts M, Olsen AM, et al. Cardiovascular safety of non-aspirin non-steroidal antiinflammatory drugs: review and position paper by the working group for Cardiovascular Pharmacotherapy of the European Society of Cardiology. Eur Heart J Cardiovasc Pharmacother. 2016;2:108-18. 
16. Upton RA, Williams RL, Kelly J, Jones RM. Naproxen pharmacokinetics in the elderly. Br J Clin Pharmacol. 1984;18:207-14.

17. Van den Ouweland FA, Jansen PA, Tan Y, Van de Putte LB, Van Ginneken CA, Gribnau FW. Pharmacokinetics of high-dosage naproxen in elderly patients. Int J Clin Pharmacol Ther Toxicol. 1988;26:143-7.

18. O'Neil CK, Hanlon JT, Marcum ZA. Adverse effects of analgesics commonly used by older adults with osteoarthritis: focus on non-opioid and opioid analgesics. Am J Geriatr Pharmacother.

2012;10:331-42.

19. Bakhriansyah M, Souverein PC, de Boer A, et al. Gastrointestinal toxicity among patients taking selective COX-2 inhibitors or conventional NSAIDs, alone or combined with proton pump inhibitors: a case-control study. Pharmacoepidemiol Drug Saf. 2017 Oct;26(10):1141-1148.

20. Avis de la Commission de Transparence - 9 novembre 2016. https://www.hassante.fr/portail/.../CT-15490_ACUPAN_PIS_RI_Avis2_CT15490.pdf

21. Naples JG, Gellad WF, Hanlon JT. The Role of Opioid Analgesics in Geriatric Pain Management. Clin Geriatr Med. 2016;32:725-735.

22. Likar R, Wittels M, Molnar M, et al. Pharmacokinetic and pharmacodynamic properties of tramadol IR and SR in elderly patients: a prospective, age-group-controlled study. Clin Ther. 2006;28:2022-39.

23. Moisset X, Trouvin AP, Tran VT, et al. Use of strong opioids in chronic non-cancer pain in adults. Evidence-based recommendations from the French Society for the Study and Treatment of Pain. Presse Med. 2016 Apr;45(4 Pt 1):447-62.

24. French HP, Smart KM, Doyle F. Prevalence of neuropathic pain in knee or hip osteoarthritis: A systematic review and meta-analysis. Semin Arthritis Rheum. 2017 Aug;47(1):1-8.

25. Rifbjerg-Madsen S, Christensen AW, Christensen R, et al. Pain and pain mechanisms in patients with inflammatory arthritis: A Danish nationwide cross-sectional DANBIO registry survey. PLoS One. 2017 Jul 7;12(7):e0180014.

26. Bouhassira D, Attal N, Alchaar $\mathrm{H}$, et al. Comparison of pain syndromes associated with nervous or somatic lesions and development of a new neuropathic pain diagnostic questionnaire (DN4). Pain. 2005 Mar;114(1-2):29-36.

27. Finnerup NB, Attal N, Haroutounian S, et al. Pharmacotherapy for neuropathic pain in adults: a systematic review and meta-analysis. Lancet Neurol. 2015 Feb;14(2):162-73.

28. Trouvin AP, Perrot S, Lloret-Linares C. Efficacy of Venlafaxine in Neuropathic Pain: A Narrative Review of Optimized Treatment. Clin Ther. 2017 Jun;39(6):1104-1122.

29. Park J, Hughes AK. Nonpharmacological approaches to the management of chronic pain in community-dwelling older adults: a review of empirical evidence. J Am Geriatr Soc. 2012;60(3):555568. 
30. Ardigo S, Herrmann FR, Moret V, et al. Hypnosis can reduce pain in hospitalized older patients: a randomized controlled study. BMC Geriatr. 2016 Jan 15;16:14.

31. Lunde LH, Nordhus IH, Pallesen S. The effectiveness of cognitive and behavioural treatment of chronic pain in the elderly: a quantitative review. J Clin Psychol Med Settings. 2009 Sep;16(3):254-62.

32. Niknejad B, Bolier R, Henderson CR Jr, et al. Association Between Psychological Interventions and Chronic Pain Outcomes in Older Adults: A Systematic Review and Meta-analysis. JAMA Intern Med. 2018 Jun 1;178(6):830-839.

33. Lin X, Huang K, Zhu G, et al. The Effects of Acupuncture on Chronic Knee Pain Due to Osteoarthritis: A Meta-Analysis. J Bone Joint Surg Am. 2016 Sep 21;98(18):1578-85.

34. Manheimer E, Cheng K, Linde K, et al. Acupuncture for peripheral joint osteoarthritis. Cochrane Database Syst Rev. 2010 Jan 20;(1):CD001977.

35. Morone NE, Greco CM, Moore CG, et al. A Mind-Body Program for Older Adults With Chronic Low Back Pain: A Randomized Clinical Trial. JAMA Intern Med. 2016 Mar;176(3):329-37.

36. Simon CB, Riley JL 3rd, Fillingim RB, et al. Age Group Comparisons of TENS Response Among Individuals With Chronic Axial Low Back Pain. J Pain. 2015 Dec;16(12):1268-1279.

37. Marcum ZA, Duncan NA, Makris UE. Pharmacotherapies in Geriatric Chronic Pain Management. Clin Geriatr Med. 2016 Nov;32(4):705-724. 
Encadré 1 : Traitements anti-neuropathiques posologies à l'initiation (à réévaluer selon les défaillances d'organes connues et les co-prescriptions)

En première intention

- Gabapentinoïdes : prégabaline à $25 \mathrm{mg} /$ jour ou de gabapentine à $100 \mathrm{mg} / \mathrm{jour}$ Paliers de doses de $25 \mathrm{mg}$ pour la pregabaline et de $100 \mathrm{mg}$ pour la gabapentine tous les 4 jours

En deuxième intention

- Antidépresseurs inhibiteurs de recapture de la serotonine et de la noradrénaline : duloxétine $30 \mathrm{mg} /$ jour ou venlafaxine $37,5 \mathrm{mg} / \mathrm{jour}$

- Tramadol : suspension buvable $(2,5 \mathrm{mg} /$ goutte $)$ permettant une titration lente

Malgré leur efficacité reconnue dans les douleurs neuropathiques, les antidépresseurs tricycliques ne sont pas recommandés en première intention en raison de leurs effets anticholinergiques importants. 